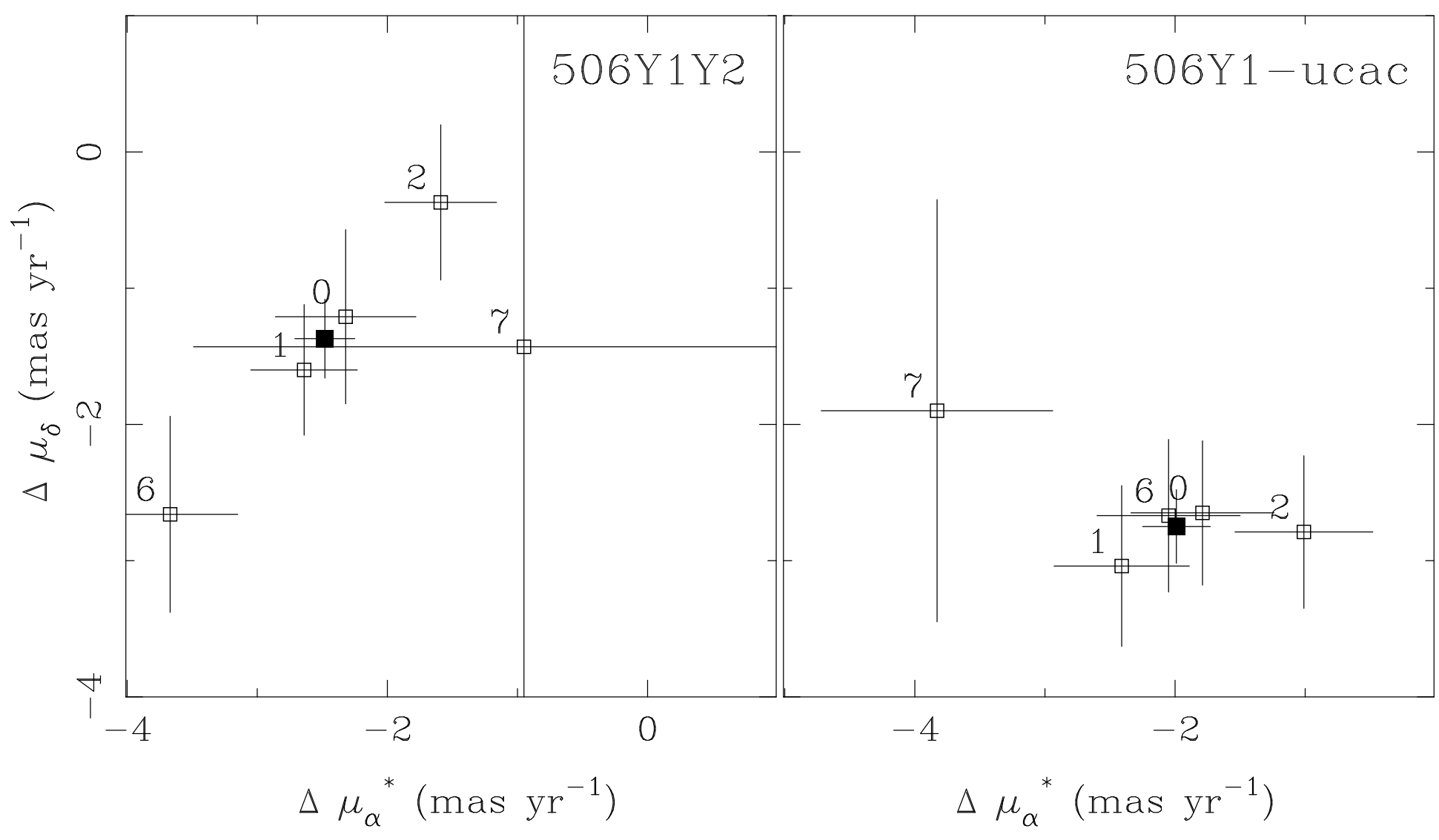



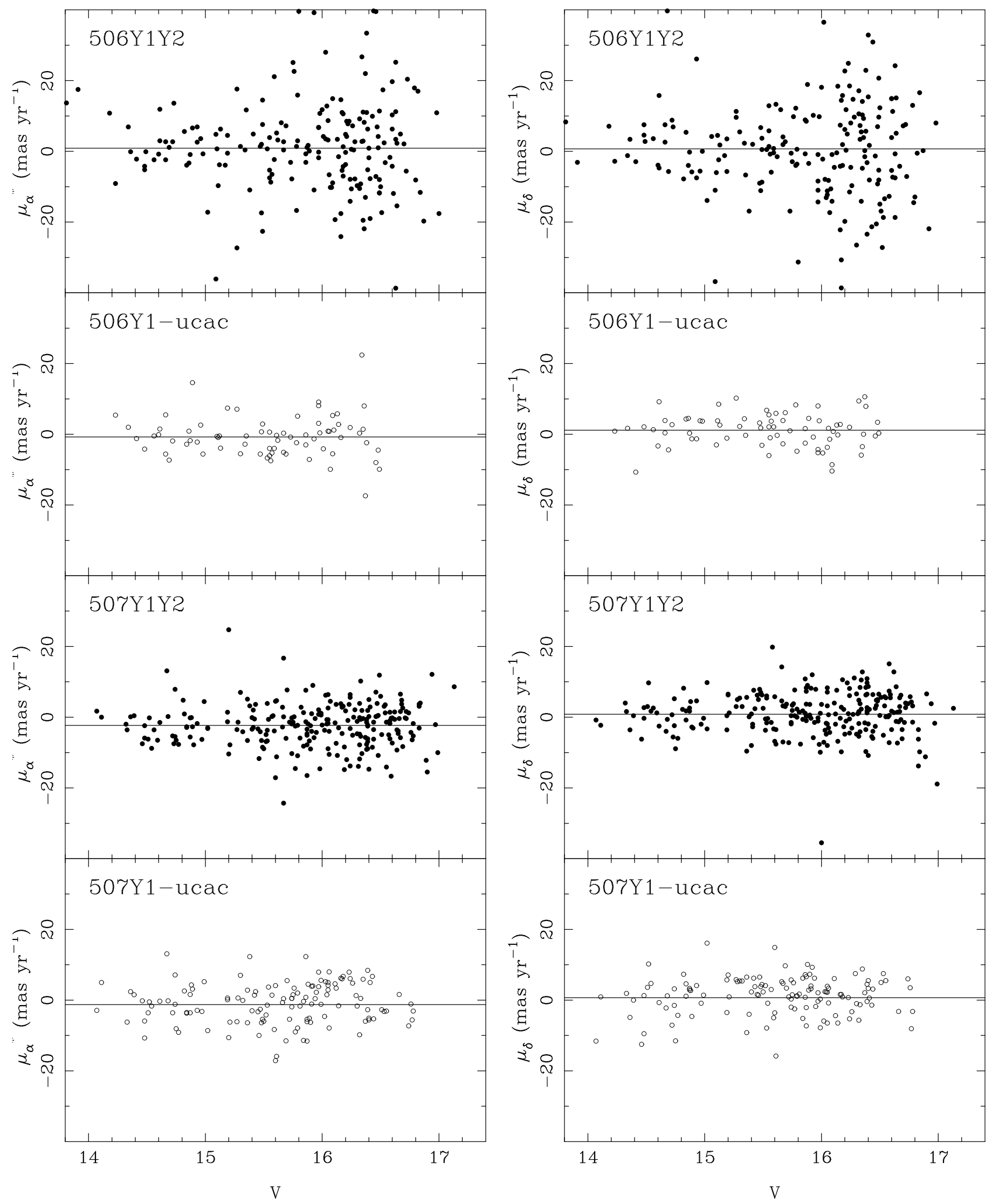
Table 2. Absolute proper-motion results

\begin{tabular}{lllrr}
\hline \hline \multirow{2}{*}{ NGC } & Solution & \multicolumn{2}{c}{$\mu_{\alpha}^{*}\left(\mathrm{mas} \mathrm{yr}^{-1}\right)$} & $\mu_{\delta}$ \\
& & $\mathrm{N}_{c l}$ \\
\hline \multirow{6}{6}{6266} & 506B1B2 & $-3.20(0.92)$ & $-0.64(0.95)$ & 197 \\
& 506Y1Y2 & $-3.11(0.51)$ & $-0.40(0.53)$ & 258 \\
& 506B1-ucac & $-3.41(1.02)$ & $-0.98(0.85)$ & 50 \\
& 506Y1-ucac & $-3.93(0.51)$ & $-1.17(0.54)$ & 122 \\
6304 & 506B1B2 & $1.36(1.41)$ & $0.23(1.37)$ & 87 \\
& 506Y1Y1 & $-1.59(0.71)$ & $-0.80(0.75)$ & 167 \\
& 506B1-ucac & $-0.36(1.10)$ & $-2.55(0.79)$ & 29 \\
& 506Y1-ucac & $-2.75(0.58)$ & $-1.61(0.54)$ & 75 \\
& 507Y1Y2 & $-3.14(0.38)$ & $-1.52(0.38)$ & 242 \\
& 507Y1-ucac & $-2.67(0.47)$ & $-2.26(0.50)$ & 130 \\
6316 & 506Y1Y2 & $-1.82(1.27)$ & $-1.03(1.47)$ & 40 \\
& 507Y1Y2 & $-2.62(0.73)$ & $-1.82(0.60)$ & 44 \\
6723 & 440B1B2 & $-1.80(0.65)$ & $-3.83(0.73)$ & 212 \\
& 440Y1B2 & $-1.01(0.53)$ & $-1.69(0.44)$ & 166 \\
& 440B1-ucac & $0.22(1.03)$ & $-2.19(1.13)$ & 87 \\
& 440Y1-ucac & $0.60(0.41)$ & $-1.80(0.78)$ & 97 \\
\hline
\end{tabular}



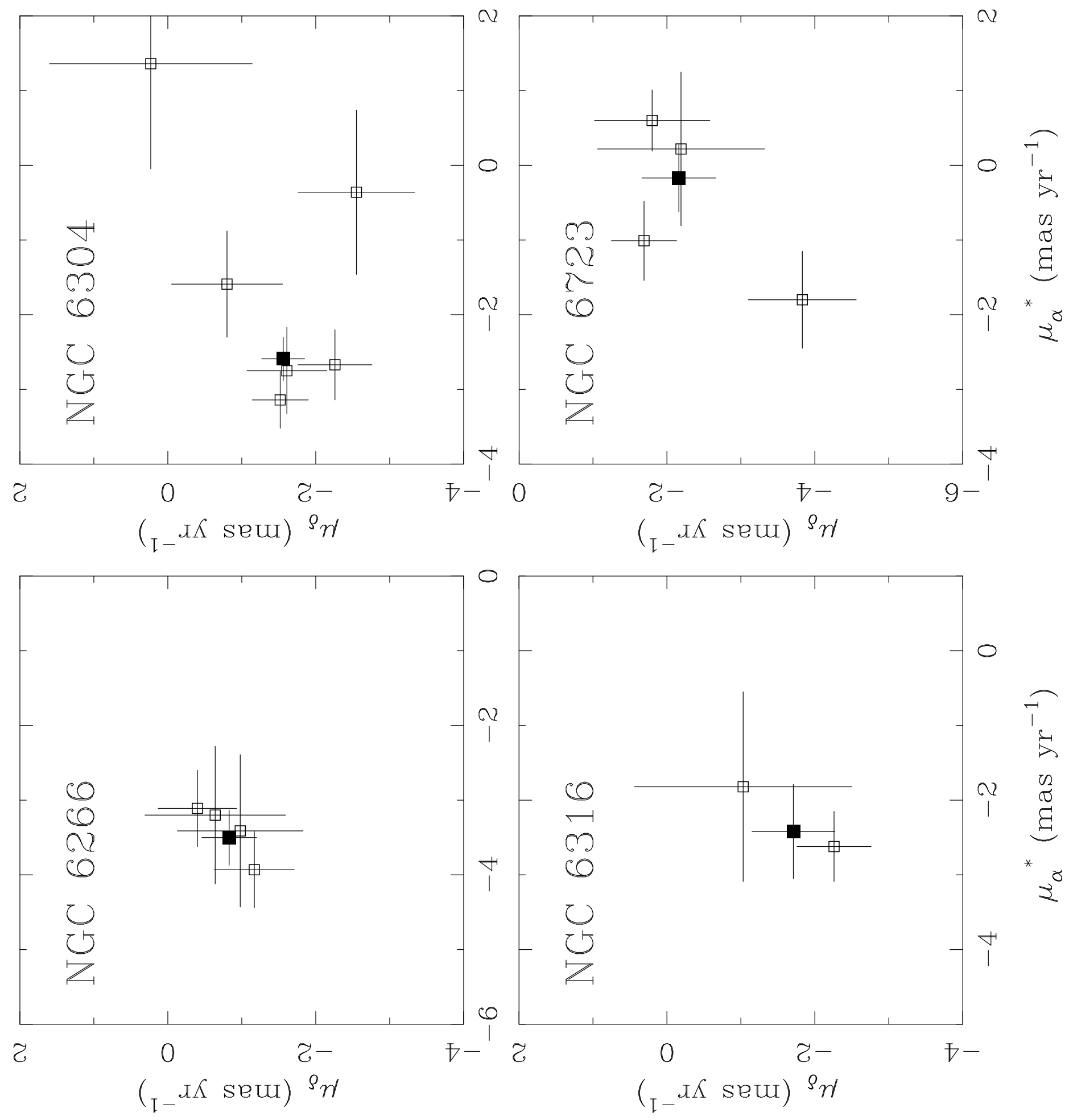
Table 3. Cluster Data

\begin{tabular}{rrrrrrr}
\hline \hline NGC & $\mathbf{l}$ & $\mathbf{b}$ & $\begin{array}{r}\mu_{\alpha}^{*} \\
\left(\mathrm{mas} \mathrm{yr}^{-1}\right)\end{array}$ & $\begin{array}{c}\mathrm{d}_{\odot} \\
(\mathrm{kpc})\end{array}$ & \multicolumn{1}{c}{$\begin{array}{c}\mathrm{V}_{\text {rad }} \\
\mathrm{km} \mathrm{s}^{-1}\end{array}$} \\
\hline \multicolumn{7}{c}{ This study } \\
6266 & 353.6 & 7.3 & $-3.50(0.37)$ & $-0.82(0.37)$ & $6.9(0.7)$ & $-70.0(1.3)$ \\
6304 & 355.8 & 5.4 & $-2.59(0.29)$ & $-1.56(0.29)$ & $6.1(0.6)$ & $-107.3(3.6)$ \\
6316 & 357.2 & 5.8 & $-2.42(0.63)$ & $-1.71(0.56)$ & $11.0(1.1)$ & $71.5(8.9)$ \\
6723 & 0.1 & -17.3 & $-0.17(0.45)$ & $-2.16(0.50)$ & $8.8(0.9)$ & $-94.5(3.6)$ \\
& & & & & & \\
6522 & 1.0 & -3.9 & $6.08(0.20)$ & $-1.83(0.20)$ & $7.8(0.8)$ & $-21.1(3.4)$ \\
6528 & 1.1 & -4.2 & $-0.35(0.23)$ & $0.27(0.26)$ & $9.1(0.9)$ & $184.9(3.8)$ \\
6553 & 5.3 & -3.0 & $2.50(0.07)$ & $5.35(0.08)$ & $5.6(0.6)$ & $-6.5(2.7)$ \\
\hline
\end{tabular}




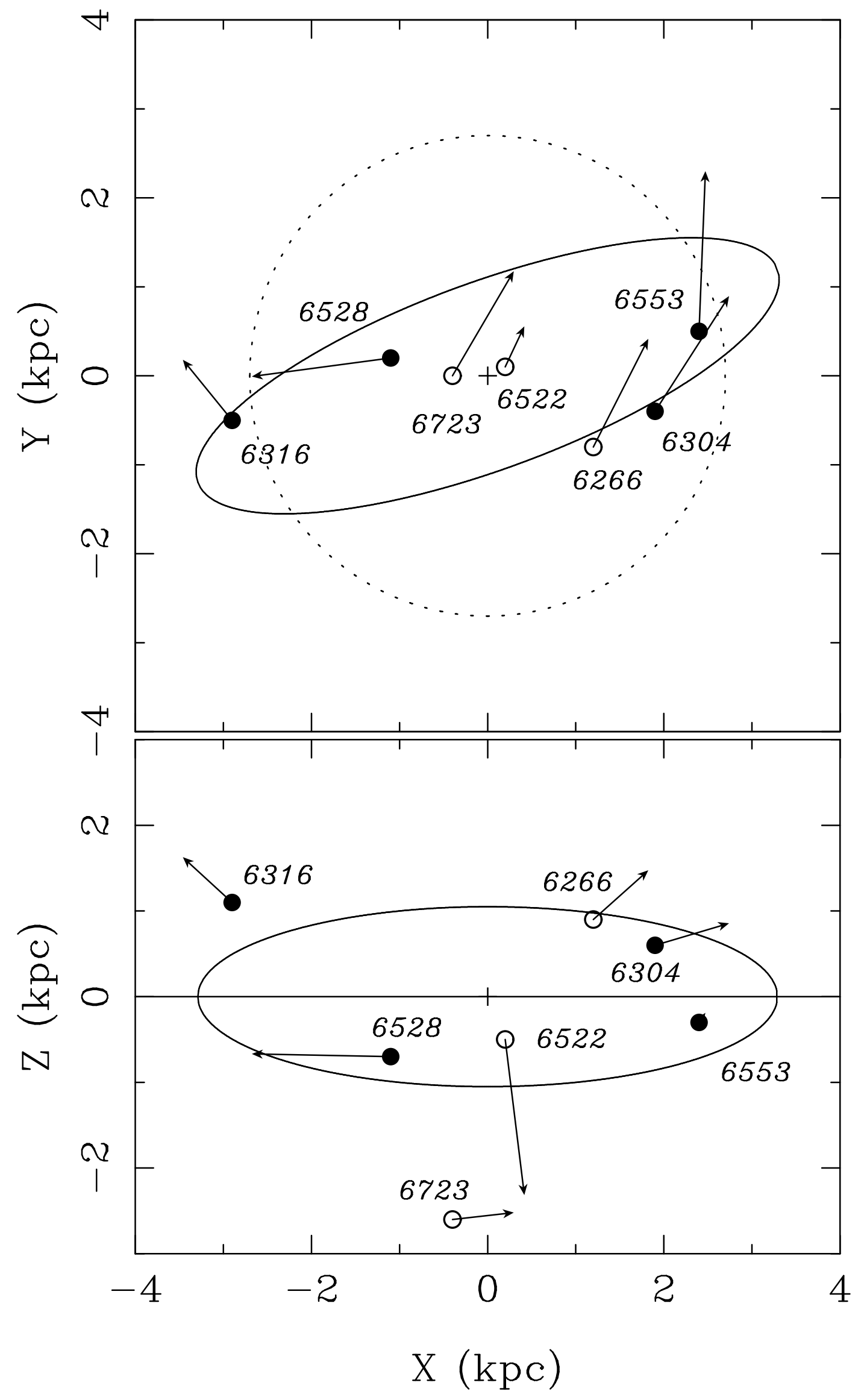




\title{
Space Velocities of Southern Globular Clusters. IV. First Results for Inner-Galaxy Clusters
}

\author{
Dana I. Dinescu ${ }^{1,2}$, Terrence M. Girard ${ }^{1}$, William F. van Altena ${ }^{1}$, and Carlos López ${ }^{3}$
}

Received —

\footnotetext{
${ }^{1}$ Astronomy Department, Yale University, P.O. Box 208101, New Haven, CT 06520-8101 (dana@astro.yale.edu, girard@astro.yale.edu, vanalten@astro.yale.edu

${ }^{2}$ Astronomical Institute of the Romanian Academy, Str. Cutitul de Argint 5, RO-75212, Bucharest 28, Romania

${ }^{3}$ Yale Southern Observatory, University of San Juan, Argentina
} 


\begin{abstract}
We have measured the absolute proper motions of four low-latitude, innerGalaxy globular clusters. These clusters are: NGC 6266 (M62), NGC 6304, NGC 6316 and NGC 6723. The proper motions are on the Hipparcos system, as no background extragalactic objects are found in these high-extinction regions. The proper-motion uncertainties range between 0.3 and 0.6 mas $\mathrm{yr}^{-1}$.

We discuss the kinematics of these clusters and of three additional bulge clusters - NGC 6522, NGC 6528 and NFC 6553 - whose proper motions with respect to bulge stars had been determined previously. We find that all of the clusters have velocities that confine them to the bulge region. Of the three metal poor clusters $([\mathrm{Fe} / \mathrm{H}]<-1.0)$, NGC 6522, and NGC 6723 have kinematics consistent with halo membership. The third cluster, NGC 6266 however, appears to belong to a rotationally-supported system. Of the four metal rich clusters $([\mathrm{Fe} / \mathrm{H}] \geq-1.0)$, NGC 6304 and NGC 6553 also have kinematics consistent with membership to a rotationally-supported system. NGC 6528 has kinematics, metallicity and mass that argue in favor of a genuine Milky-Way bar cluster. NGC 6316's kinematics indicate membership to a hotter system than the bar.
\end{abstract}

Subject headings: (Galaxy:) globular clusters: individual (NGC 6266, NGC 6304, NGC 6316, NGC 6723 - Galaxy: bulge — astrometry -)

\title{
1. Introduction
}

Our Galaxy's inner regions are a challenge for Galactic structure studies, as they consist of a superposition of stellar populations of various spatial, physical and kinematical properties, all of which peak in density in these regions. In order to understand the stellar 
populations of the inner Galaxy, one desires a tracer population that has, at the least, reliable measurements of distances, radial velocities, proper motions and metallicities, and, ideally, detailed chemical abundances and ages. Globular clusters fulfill these requirements well. Their only drawback is their small number, when compared to that of field stars.

There is still the question of whether the clusters are representative of the Galaxy's field stellar population. Many recent studies point to the fact that the globular-cluster population does resemble in many ways the old stellar field component. Based on radial velocities and metallicities it has been shown that globulars can be divided into thick disk and halo components (Zinn 1985, Armandroff 1989). Later, Minniti (1995) studied the density distribution of metal rich clusters in the bulge region $\left(r_{G C} \leq 3 \mathrm{kpc}\right)^{1}$, and he concluded that most of these clusters belong to the bulge system rather than the disk. More recent distance, radial-velocity, and metallicity measurements of clusters in the bulge region together with more detailed analyses (Barbuy, Bica, \& Ortolani 1998, Côté 1999, Heitsch \& Richtler 1999; hereafter HR99, Burkert \& Smith 1997; hereafter BS97) suggest that some clusters may form a bar system that resembles the Milky Way bar (see Gerhard 2002 for a review of observations and characteristics of the Galactic bar).

Regarding clusters outside the bulge region but within the solar circle, Zinn (1996) shows that intermediate metal-poor clusters $(-1.8<[\mathrm{Fe} / \mathrm{H}]<-0.8)$ have significant rotation $\left(V_{\text {rot }} \sim 60 \mathrm{~km} \mathrm{~s}^{-1}\right)$ which is however, lower than that of the thick disk. This analysis included radial-velocity data alone and the typical Frenk \& White (1980) solution, that can determine a mean rotation of a pre-selected group of clusters.

Adding tangential velocities as a piece of crucial information, Cudworth \& Hanson

\footnotetext{
${ }^{1}$ Here, $r_{G C}$ represents the Galactocentric radius in a spherical coordinate system, and $R_{G C}$ the Galactocentric distance in a cylindrical coordinate system.
} 
(1993) and later, Dinescu, Girard \& van Altena (1999; Paper III) have unambiguously shown that there is a metal-weak thick disk component in the cluster population, much like that seen in the local field stellar population (see Beers et al. 2002 for the most recent study). And, the inner-halo rotation detected by Zinn (1996), was also found in the studies that included tangential velocities (Paper III). This rotation is qualitatively consistent with the flattened shape of the inner halo as determined from field stars (e.g., Hartwick 1987, Chiba \& Beers 2001, Siegel et al. 2002). To summarize, the globular-cluster system of our Galaxy appears to resemble the old stellar field component.

We set out to measure tangential velocities (i. e., absolute proper motions) for a sample of fifteen clusters (Dinescu, Girard, \& van Altena 2002) primarily located within the solar circle, in order to more accurately assign clusters to the components of the inner Galaxy, and to better understand these components as regions where old and massive stellar systems were produced/captured.

In this paper we present the first results for four globular clusters located in the bulge region: NGC 6266 (M 62), NGC 6304, NGC 6316, and NGC 6723. We discuss the kinematics of our four globular clusters and of three additional clusters (NGC 6522, NGC 6528, and NGC 6553) located in the bulge region that have previous proper-motion measurements, in light of the Galactic components seen in the bulge region.

The proper-motion measurements are described in Section 2, the velocity results are presented in Section 3, and discussed in Section 4, and a brief summary is presented in Section 5 . 


\section{Measurements}

This project is part of the continuation of the Southern Proper Motion Program (SPM, Platais et al. 1998), and a low-latitude supplement to the previous set of fifteen high-latitude globular clusters measured by our group (Dinescu et al. 1997, 1999, Paper I and II respectively). SPM plates are taken with the 50-cm double astrograph at Cesco Observatory in El Leoncito, Argentina (plate scale $=55.1$ " $/ \mathrm{mm}$ ). The first-epoch survey, covering the sky south of declination $-17^{\circ}$, was completed in the early 1970 's. The second-epoch survey was made in the 1990's, but after completing roughly one third of the intended coverage, Kodak discontinued production of the necessary 103a emulsion plates. The remainder of the second-epoch survey will be obtained with a $4 \mathrm{Kx} 4 \mathrm{~K}$ CCD camera which has recently been installed on the astrograph.

The four clusters presented here lie within SPM fields which have both first and second epoch photographic plates. The results in this paper are based on these plates, and on the UCAC1 catalog (Zacharias et al. 2000, hereafter Z00). Usually, there are 2 plate pairs (blue: $103 \mathrm{aO}$, and visual: $103 \mathrm{aG}+$ OG515 filter) per SPM field, which cover an area of 6.3x6.3. Each SPM plate contains two exposures: a two-hour exposure, which reaches to about $V=18$, and an offset two-minute exposure, which allows a tie-in to bright reference stars. During both exposures, an objective grating is used, which produces a series of diffraction images on either side of the central zero-order image.

The program clusters were selected based on the limitations imposed by the SPM survey. Thus, they are located in the southern sky, and within distances of $11 \mathrm{kpc}$ from the Sun. A few additional clusters at larger distances are in our program list because they happen to be on the same SPM field with one or two more nearby clusters.

Since detailed descriptions of the measurement process and reduction procedures are given in Papers I and II, we will only outline here the most important aspects. 
The photographic plates are scanned with the Yale PDS microdensitometer, in an object-by-object mode, at a pixel size of 12.7 microns. We prepare an input catalog that contains all Hipparcos and Tycho (ESA 1997) stars, 3000 faint field stars selected from the USNO-A2.0 catalog (Monet et al. 1998) in the magnitude range 15 to $17, \sim 150$ stars from the GSC 1.1 catalog (Lasker et al. 1990) with magnitudes fainter than those of the Tycho stars $(V=11-14)$, and cluster stars. Hipparcos stars provide the correction to absolute proper motion, while Tycho and GSC stars assure a good range in the magnitude of various diffraction orders in order to model magnitude-dependent systematics. For all of these relatively bright stars, we measure long and short-exposure images, and diffraction images.

The faint stars from the USNO-A2.0 catalog allow a well-determined plate model (see Paper I), and for these stars we measure only the long-exposure, central order image. The list of cluster stars is determined using the software package Sextractor (Bertin \& Arnouts 1996) from a preliminary scan in the cluster region of the best quality plate. Cluster stars are selected in a region of radius 2-3 times the half-mass radius as taken from Harris (1996; hereafter H96). Typically, this radius is 3-4 arcmin, with the very central 1-2 arcmin totally unusable because of crowding. For the cluster stars we also measure only the long-exposure, central order image.

The dominant systematic effect in these proper-motion measurements is a magnitudedependent shift in the image position owed to the nonlinear response of the photographic emulsions and asymmetric image profiles caused by guiding errors during long exposures, imperfections in the optical system, polar misalignment of the telescope, etc. We call this bias "magnitude equation", and we caution that it is present in virtually all photographic plate material. For our plate material for instance, over a range of 6 magnitudes (typical globular cluster stars have $V \geq 15$, and Hipparcos stars have $V \sim 9$ ), magnitude equation 
can produce deviations of 10-15 mas $\mathrm{yr}^{-1}$ (Girard et al. 1998). Note that, at a distance of 6 $\mathrm{kpc}, 1$ mas yr${ }^{-1}$ corresponds to $28 \mathrm{~km} \mathrm{~s}^{-1}$. We use the diffraction images in order to correct for magnitude equation according to the prescription described in Girard et al. (1998). In short, in absence of magnitude equation, each diffraction-order pair of images would be symmetrically displaced relative to the central order. Magnitude equation produces a deviation of the position of the central order image from the position expected from the geometry of the diffraction images, as the central-order image is 4 magnitudes brighter than the diffraction images. Thus, by measuring a sufficient number of stars whose central and diffraction images are separable, we are able to deduce the exact form of the magnitude equation on each SPM plate, and correct for it (see details and tests of this procedure in Girard et al. 1998).

The magnitude equation correction is of significant size, and therefore, at the bright end, there is a non-negligible uncertainty in it. Thus, in the correction to absolute proper motion, we will use only Hipparcos stars fainter than $V=8$, to avoid residual magnitude equation in our proper motions. Also, the blue plates used in this study have a poorer determination of the magnitude equation than that of the visual plates, because the "blue" images are less sharp than the "visual" ones. This is due to the fact that the blue plates are taken with no filter, and atmospheric seeing is generally poorer in the blue than in the visual passband. On some fields, in the blue passband only, we had to use the second-order diffraction images, because the first-order images were blended with the central order.

We also obtain photographic photometry by calibrating the instrumental magnitudes with Tycho magnitudes, and with already published CCD and/or photoelectric photometry in the cluster region. This latter photometry ensures a good calibration at the faint end. Our photographic magnitudes have uncertainties of 0.15-0.25 mags. There were two overlapping SPM fields for clusters NGC 6266, NGC 6304, and NGC 6316. For these two 
fields, we have used CCD photometry from Brocato et al. (1996) (NGC 6266), Rosenberg et al. (2000) (NGC 6266, and NGC 6304), and Ortolani et al. (2000) (NGC 6304). For the SPM field containing NGC 6723, we have used photoelectric photometry from Alvarado et al. (1994) and CCD photometry from Rosenberg et al. (2000).

\section{Results}

\subsection{Proper Motions}

Table 1 summarizes our plate-pair solutions. We have also used the UCAC1 positions in combination with our first-epoch plates, because they provide a longer baseline $(\sim 30$ years; mean epoch 1999) than the SPM plates alone. However, UCAC1 is less deep $(R \leq 16)$ than the SPM plate material, and therefore solutions including this catalog miss a number of faint cluster stars. The denomination of each plate solution in Table 1 is as follows: the number represents the SPM field number; B1, Y1, represent the blue, and visual first-epoch plate respectively, and similarly B2, Y2 refer to the second-epoch plates; ucac represents the corresponding SPM area selected from the UCAC1 catalog.

From a full plate solution that uses a large number of reference stars $(\sim 3000)$, with a denser ring of stars around the cluster (Paper I and II), we obtain relative proper motions for all of the stars/images. The difference between the Hipparcos absolute proper motions and the relative proper motions obtained in our solution, represents the correction to absolute proper motion to be applied to the relative proper motion of the cluster stars. We calculate this proper-motion difference for each SPM grating-image order of the Hipparcos stars. We plot these differences as a function of color, magnitude and position on the plate, to inspect for residual systematics. For each image order, we calculate the average and the scatter of these differences using probability plots (see Paper I and II). The final 
correction to absolute proper motion is a weighted mean of the correction given by each image order. The weights are given by the internal scatter within each image order (see also Paper II for cluster M4). In some cases we simply discard image orders that we know are erroneous (poor image quality, uncertain magnitude-equation correction). The number of Hipparcos stars in each image order ranges between 30 and 90. In Figure 1 we show, as an example, the correction to absolute proper motion for plate-pair solutions 506Y1Y2 and 506y1-ucac (left and right panel respectively; $\mu_{\alpha}^{*}=\mu_{\alpha} \cos \delta$ ). Each point represents the average of a given image order and is labeled as follows: 0 - long-exposure central order, 1 long-exposure first order, 2 - long-exposure second order, 6 - short-exposure central order, 7 - short-exposure first order, and 8 - short-exposure second-order (The image order 8 is not shown here because it is a very poor measurement). The error bars are uncertainties in the averages as given by the internal error estimate of each image order. The weighted mean of all image orders are also shown in the figure, with the dark symbols. Overall, the 506Y1-ucac measurement appears to be better than the 506Y1Y2 from the scatter and the formal errors in each image order. However, the 506Y1-ucac measurements are not independent (as the SPM ones are), because for each image order on the first-epoch SPM plate there is a corresponding image-order measurement on the second epoch, but only one UCAC1 position.

The uncertainty in the mean cluster motion is dominated by the image centering error from the PDS scans and a relatively small number of cluster stars. We plot the centering error as a function of magnitude, and select those stars that follow a known sequence as determined from stars in the uncrowded region of the plate; a centering-error upper limit

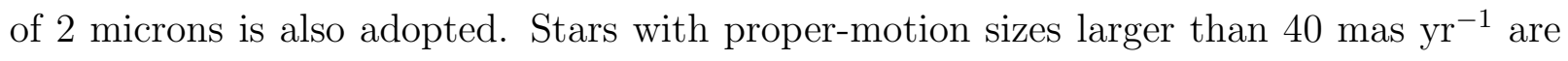
excluded. The average relative cluster proper motion is a centering-error weighted mean. We have also used probability plots to estimate this quantity, and we found that it is similar to the weighted mean within the uncertainties. The field star contamination does not affect 
our mean cluster proper motion for the following reasons: 1) the cluster area is a very small region, where $10-15 \%$ of the stars measured in the cluster area may belong to the field (this estimate is based on the stellar density in the ring of reference stars that surrounds the cluster, see also Paper I), and 2) the field proper-motion dispersion is large $(\sim 10$ mas $\mathrm{yr}^{-1}$ ) in the magnitude range $V=14-18$ of the reference stars; thus, the potential of systematically affecting our estimate of the mean cluster motion is negligible. In Figure 2 we show relative proper motions in the region of NGC 6304 as a function of magnitude, for visual-plate solutions in SPM fields 506 and 507. The stars shown are those trimmed in centering-error magnitude space. The horizontal lines represent the centering-error weighted means. Using cluster stars, whose proper-motion dispersion reflects the measurement error only, and assuming that the positional uncertainty on the SPM plates is the same at both epoch, we can estimate the positional uncertainty for UCAC1 stars in the magnitude range $14 \leq V \leq 17$. From our Y plate-pair solutions that have the best quality images on SPM fields 506 and 507, we obtain a positional uncertainty between 98 and 110 mas for UCAC1 stars in NGC 6304. These values are larger than the standard error of UCAC1 (Z00) at $R=16: 70$ mas. This is perhaps due to crowding/blending in the cluster region due to the 100 "/mm plate scale of the US Naval Observatory Twin Astrograph.

The absolute proper motion is the sum of the relative cluster proper motion and the correction to absolute as given by the Hipparcos stars (also called zero point). Uncertainties

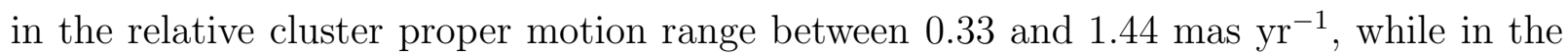

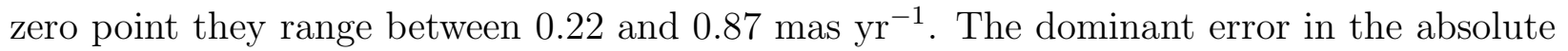
proper-motion uncertainty is that given by the relative cluster motion. In Table 2 we summarize our absolute proper motions for each cluster and plate solution; we also specify the number of cluster stars used in each solution. In Figure 3 we plot the results from Table 2, on a single scale for comparison purposes. 
We note that some data points in Fig. 3 are not strictly independent, because of the use of the same plate in two plate-pair solutions (Table 1). This has been taken into account when we estimate the final absolute proper motion and associated uncertainty. We do so by determining relative mean positions of each cluster from the absolute proper-motion measures (see Table 2) at the three different epochs, as follows. We adopt an arbitrary nominal position for the UCAC1 epoch. For each passband, we predict a position at the 1st-SPM epoch using the SPM-ucac proper motions. Then, using the SPM-only solution, we predict the 2nd-SPM epoch positions. From the proper-motion uncertainty, and the number of cluster stars used in each plate-pair solution we are able to obtain positional uncertainties for the mean cluster position at a given epoch. Then, we determine a (position-uncertainty) weighted fit of the mean cluster position as a function of time. The slope and its formal error from the fit yield the final absolute proper motion and its corresponding uncertainty.

For NGC 6266, there are five independent position data points (see Table 1). We obtain $\mu_{\alpha}^{*}=-3.50 \pm 0.37$ and $\mu_{\delta}=-0.82 \pm 0.37$ mas $\mathrm{yr}^{-1}$ for NGC 6266. For NGC 6304, using seven independent mean cluster positions (Table 1), we obtain: $\mu_{\alpha}^{*}=-2.59 \pm 0.29$

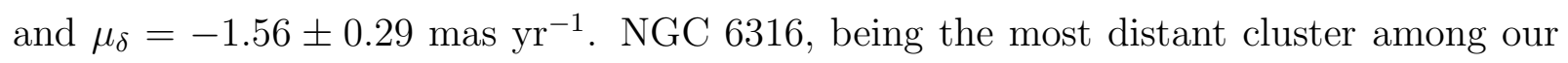

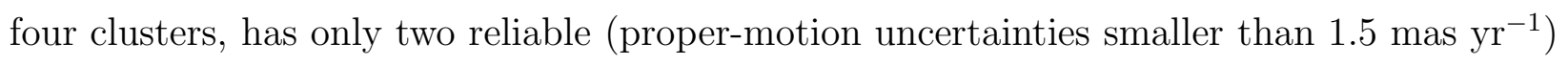
measurements provided by the visual plate pairs (Table 1,2). These two measurements are independent, and our final proper motion is a weighted mean of the two values from Table

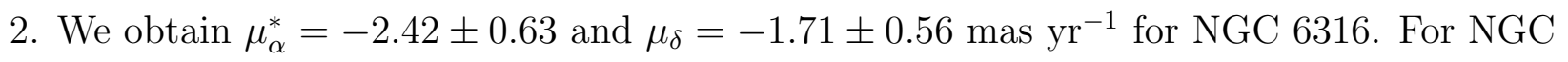
6723, we apply the same procedure as for NGC 6266, and 6304. According to the plate-pair solutions (Table 1) there are five mean cluster positions, of which those at the second SPM epoch are not independent, because of the mixed 440Y1B2 plate solution. We scale up our formal proper-motion uncertainties by the factor $\sqrt{\left(N_{p t s ; a l l}-N_{\text {fit }}\right) /\left(N_{p t s ; i n d e p .}-N_{\text {fit }}\right)}=$ 1.22 , where $N_{\text {fit }}=2$. We thus obtain $\mu_{\alpha}^{*}=-0.17 \pm 0.45$ and $\mu_{\delta}=-2.16 \pm 0.50 \mathrm{mas} \mathrm{yr}^{-1}$ for NGC 6723. The final absolute proper motions are represented with filled symbols in 
Fig. 3.

\subsection{Velocities}

In addition to our four clusters, we have selected three clusters located in the bulge region that have previous proper-motion measurements. For these, the correction to an inertial reference frame is based upon the assumption that the reference stars are bulge stars, all located at the same (known) distance, and their velocity with respect to the Galactic rest frame is, on the mean, zero. These clusters are NGC 6522 (Terndrup et al. 1998), NGC 6528 (Feltzing \& Johnson 2002), and NGC 6553 (Zoccali et al. 2001). The latter two studies are based on HST data. The Terndrup et al. (1998) study has a detailed description of the assumptions and biases of this method. An important assumption is that of only a small deviation from the Galactic Center (GC) in latitude and, most importantly, in longitude (see Discussion Section for NGC 6553). Here, we will rederive their space velocities according to the published proper motions.

In Table 3 we summarize the basic parameters for deriving space velocities. Distances and heliocentric radial velocities are from H96. The numbers in parentheses represent the uncertainties. We adopt a nominal distance uncertainty of $10 \%$ of the distance. We use the Solar peculiar motion $\left(U_{\odot}, V_{\odot}, W_{\odot}\right)=(-11.0,14.0,7.5) \mathrm{km} \mathrm{s}^{-1}$ (Ratnatunga, Bahcall, \& Casertano 1989), where $U$ is positive outward from the GC, $V$ is positive toward Galactic rotation, and $W$ is positive toward the north Galactic pole. The Sun is located at (X, Y, Z) $=(8.0,0.0,0.0) \mathrm{kpc}$, and the rotation velocity of the local standard of rest is taken to be $V_{L S R}=220.0 \mathrm{~km} \mathrm{~s}^{-1}$. In what follows we will use $V^{\prime}=V+V_{L S R}$, and velocity components in a cylindrical coordinate system of $\Pi$ (positive outward from GC) and $\Theta$ (positive toward Galactic rotation). For clusters located at small GC distances (i.e., $R_{G C}$ ), the cylindrical coordinate system is no longer appropriate, and $\Pi$ and $\Theta$ components can be misleading. 
In these cases, the Cartesian system in the Galactic rest frame $\left(U, V^{\prime}, W\right)$ is more suitable. We will come back to this issue when we discuss the kinematics of each cluster.

Table 4 lists the (X, Y, Z) coordinates, the velocity components, and the rotation velocity expected from a solid body that has an angular velocity equal to the pattern speed

of the Milky Way bar at the location of the cluster $\left(\Omega_{b}=60 \mathrm{~km} \mathrm{~s}^{-1} \mathrm{kpc}^{-1}\right.$, see the following Section).

\section{Discussion}

All of the clusters under discussion reside within $\sim 3 \mathrm{kpc}$ of the GC (Tab. 4). This is a very complex region dominated by a bar/bulge where a traditional kinematical distinction between the disk and the bar/bulge may not be as meaningful as in more distant regions from the GC, where disk and halo populations for instance are easily separated. Dynamical criteria allow the existence of a kinematical disk only outside the corotation radius (see e. g., Pfenniger \& Friedli 1991). N-body simulations show that bars form easily as a gravitational instability in a cold disk, and, in order to survive, they have to rotate (Pfenniger 1999). Bars then, can be regarded as "thickened" elliptical disks. They preserve the "memory" of the original rotating axisymmetric disk, and therefore one may be able to kinematically distinguish between the bar/bulge and a pressure-supported system such as the halo. Presumably, a halo particle will remain a halo particle, while a disk particle will become a bar/bulge particle inside the corotation radius. In N-body simulations, there is evidence of some resonant halo stars trapped in the bar, in particular at corotation (Athanassoula 2002); however, for our study that considers very small numbers, we believe that it is unlikely to find a halo globular cluster on a resonant orbit with the Galactic bar.

A separation between bar and bulge is more ambiguous given the variety of processes 
that may contribute to bulge formation such as bar growth, bar dissolution, accretion and/or merging with satellites.

In our Galaxy, evidence for a bar comes from observations of motions of atomic and molecular gas, from near-infrared photometry (NIR), IRAS sources and clump stars counts, and stellar kinematics (see e.g., Gerhard 1996 for a review). The characteristics of the Milky Way bar are summarized in Gerhard (2002). The bar has a semimajor axis a $=3.1-3.5$ kpc, an axis ratio of 10:3-4:3, and an orientation angle (angle between the semimajor axis and the Sun-GC line) of $\phi_{b a r}=15^{\circ}-35^{\circ}$. The pattern speed is $\Omega_{b}=50-60 \mathrm{~km}$ $\mathrm{s}^{-1} \mathrm{kpc}^{-1}$, and the corresponding corotation radius is $R_{C R}=3.3-5 \mathrm{kpc}$. The Galactic bar seems to be a fairly massive system $\left(\mathrm{M}_{b a r} \sim 10^{10} \mathrm{M}_{\odot}\right.$; Stanek et al. 1997, Weiner \& Sellwood 1999), of an age of up to $\sim 6-7$ Gyr (as determined from Carbon stars, Cole \& Weinberg 2002 and references therein). Given these considerations, can we speak of a globular-cluster bar system? Were these clusters trapped into bar-like orbits from an initially disk-like configuration, as dissipationless N-body simulations show? Or, were these clusters produced within the bar, if the bar formed from gas rather than from a stellar distribution? BS97 argued that low-mass (i.e. integrated absolute magnitude $\mathrm{M}_{V} \geq-7.9$ ), metal-rich $([\mathrm{Fe} / \mathrm{H}]>-0.8)$ clusters show spatial distribution and kinematics - as derived from radial velocities - consistent with those of the Milky Way bar (see also Côté 1999 and references therein). Given the large mass of the bar, and its upper age limit, it is quite conceivable that low-mass, metal-rich clusters formed within the bar. Dating the clusters suggested by BS97 as belonging to a bar-like configuration could be very instructive.

In light of the above, we proceed to discuss the results for the seven clusters under study, without attempting a detailed orbit integration that we defer to a future paper.

In Table 5 we list the cluster metallicity $[\mathrm{Fe} / \mathrm{H}]$, integrated absolute magnitude $\mathrm{M}_{V}$ (H96), total velocity, escape velocity from the bulge, and orbit inclination. The orbit 
inclination is $\Psi=90^{\circ}-\sin ^{-1}\left(\mathrm{~L}_{z} / \mathrm{L}\right)$, where $\mathrm{L}_{z}$ is the angular momentum perpendicular to the Galactic disk, and $\mathrm{L}$ is the total angular momentum, at the present time. In contrast to Paper III, this is not an orbit-averaged quantity, but an instantaneous one. The bulge potential is the Hernquist spheroid described in Johnston, Spergel \& Hernquist (1995; JSH95). This potential is a simple description of the inner Galaxy, and it is used only to estimate the escape velocity and not to derive orbits. As total velocities are smaller than escape velocities, all of the clusters are confined to the bulge region.

In Figure 4 we show the locations and motions of the clusters in the Galactic plane (top panel) and perpendicular to the Galactic plane (bottom panel). The open symbols represent the metal poor clusters, the filled symbols the metal rich ones (see following subsections). The dashed line represents the bulge region; it has a radius of $2.7 \mathrm{kpc}$, which corresponds to an angle of $20^{\circ}$ on the sky from the GC (from COBE and IRAS maps, and $R_{\odot}=8.0$ kpc). The ellipse represents the bar. Its characteristics are those determined by Bissantz \& Gerhard (2002) from a model that is constrained by the dereddened COBE/DIRBE L-band luminosity distribution and by the distribution of red clump stars in some bulge fields. The bar has a semimajor axis of $3.5 \mathrm{kpc}$, an axis ratio of 10:3:3, and an orientation angle of $20^{\circ}$.

\subsection{Metal Poor $([\mathrm{Fe} / \mathrm{H}]<-1.0)$ Clusters}

NGC 6522 and NGC 6723 have locations and velocity components that produce highly inclined orbits (Tab. 5). Their kinematics suggest these clusters are members of the halo system. Their low metallicities are in agreement with this assignment (e. g., Armandroff 1989). We note that, within the uncertainties of their distance estimates, NGC 6723 and NGC 6522 might be positioned on either the far side or near side of the GC, in which case the velocity components $\Pi$ and $\Theta$ change signs. Thus retrograde orbits become prograde and vice versa; however this does not alter the large inclination of their orbits. In other 
words, the sense of rotation with respect to the disk is of little significance when orbits are nearly polar (Tab. 5).

NGC 6266 has a large rotation velocity, while the other two components are relatively low (Tab. 4). This velocity vector, combined with a galactocentric distance $R_{G C}=1.4 \mathrm{kpc}$ (Tab. 4) indicate that NGC 6266 belongs to a rotationally supported system. Its rotation velocity is somewhat larger than that derived for a solid body rotating with the Galactic bar's pattern speed (Tab. 4). As the notion of a kinematical stellar disk is inappropriate at these galactocentric distances, we can think of NGC 6266 as a cluster that belonged to a disk system before the bar formed, rather than to a pressure-supported system such as the halo. In this sense, NGC 6266 reminds us of thick-disk, metal-poor clusters such as NGC 6626, NGC 6752, and perhaps NGC 6254 (see Paper III). These clusters represent the metal-weak thick disk (MWTD), found to be very prominent in the field stellar component within $1 \mathrm{kpc}$ of the Sun (Beers et al. 2002 find a fraction of 30-40\% MWTD at metallicities $<-1.0$ ). The novelty provided by the clusters is that this component can be probed at smaller galactocentric radii than local samples.

\subsection{Metal Rich $([\mathrm{Fe} / \mathrm{H}]>-1.0)$ Clusters}

Based on spatial distribution, radial velocities and masses, BS97 have proposed a new classification of clusters with $[\mathrm{Fe} / \mathrm{H}]>-0.8$. The high-mass system $\left(\mathrm{M}_{V}<-7.9\right)$ is thought to be representative of the bulge. It has an angular velocity $\omega=15 \mathrm{~km} \mathrm{~s}^{-1} \mathrm{kpc}^{-1}$, and a line-of-sight velocity dispersion $\sigma_{l o s}=56 \mathrm{~km} \mathrm{~s}^{-1}$, assuming a solid-body rotation in a Frenk \& White (1980) type solution. The low-mass system $\left(\mathrm{M}_{V} \geq-7.9\right)$ is a sum of two components: a bar-like component, and a thick-disk-like component. The bar-like component has an angular velocity $\left(\sim 59 \mathrm{~km} \mathrm{~s}^{-1} \mathrm{kpc}^{-1}\right)$ similar to traditional values of Milky Way bar's pattern speed, and a $\sigma_{l o s}=55 \mathrm{~km} \mathrm{~s}^{-1}$. The thick-disk-like component 
resides at $R_{G C} \sim 5 \mathrm{kpc}$, and it has typical thick-disk kinematics (see Table 1 in BS97).

According to BS97, by mass and metallicity, NGC 6316 is a bulge cluster (Table 5). If we use the angular velocity of the bulge globular-cluster system defined in BS97 to predict the rotation velocity at NGC 6316's location, we obtain that NGC 6316's retrograde velocity is at $2.2 \sigma$ of that predicted by the solid body. To estimate $\sigma$ we have used the uncertainty in the $\Theta$ component (Tab. 4) and we have taken the velocity dispersion along $\Theta$ to be equal to the line-of-sight velocity dispersion derived from the solid-body solution for all of the candidate bar clusters in BS97. The constant-rotation velocity solution in BS97 places NGC 6316 within 1.6 $\sigma$ of the mean of the globular-cluster bulge system defined by BS97, as the line-of-sight velocity dispersion is $\sim 70 \mathrm{~km} \mathrm{~s}^{-1}$. A similar comparison with the BS97 bar system, places NGC 6316 at $4.3 \sigma$ from the rotation velocity expected from the solid body. Thus, according to its velocity, NGC 6316 can be thought of as a bulge cluster rather than a bar cluster, and this is in agreement with its classification according to metallicity and mass.

Both NGC 6304 and NGC 6553 have large rotation velocities and low to moderate radial and vertical velocity components (Tab. 4). The $\Theta$ components are somewhat larger than the rotation velocities expected from a solid body that has an angular velocity equal to the Galactic bar's pattern speed (Tab. 4). The large $\Theta / V^{\prime}$-velocity component of NGC 6553 may be due to the assumption that the reference stars (bulge stars) in the proper-motion solution have zero velocity (see Section 3.1). The Galactic longitude of this cluster is larger than that of NGC 6522, and 6528, therefore this assumption may not be correct. In fact, at a longitude of $\sim 5^{\circ}$ (Table 3 ), the bulge stars are expected to have a rotation of $45 \mathrm{~km} \mathrm{~s}^{-1}$ (Tiede \& Terndrup 1999 measure a projected mean rotation of $9 \mathrm{~km}$ $\left.\mathrm{s}^{-1} \mathrm{deg}^{-1}\right)$. Thus, the rotation-velocity component should, more likely, be $V^{\prime} \sim 180 \mathrm{~km} \mathrm{~s}^{-1}$, and correspondingly, $\Theta=175 \mathrm{~km} \mathrm{~s}^{-1}$, a value that is much closer to NGC 6304's rotation 
velocity. Masses and metallicities assign these clusters to the bar system of BS97 (NGC 6553 is slightly more massive than the BS97 limit; see Tab. 5). Their kinematics, at face value, are not inconsistent with bar membership, especially if we must abandon the idea of disk-like orbits inside the corotation radius. These clusters could very well be members of the former disk/thick-disk transformed into a bar in the inner regions. They can be thought of as the extension of the 5-kpc thick disk clusters in BS97 into the inner Galactic regions. What is intriguing is that NGC 6266, a massive, metal-poor cluster, is kinematically very similar to NGC 6304 and NGC 6553 (Tab. 4, 5; Fig. 4). The slight difference is that NGC 6266, while currently located farther from the Galactic plane than NGC 6304 and NGC 6553 (Fig. 4), moves away from the plane with the largest velocity of the three clusters (Tab. 4). Strictly speaking, this implies that NGC 6304 and 6553 are in a more flattened system than NGC 6266.

NGC 6528 qualifies as a bar cluster according to BS97 (Tab. 5). Its $\Theta$ velocity component is in very good agreement with the rotation of a solid body that has the Galactic bar's pattern speed (Tab. 4). The very low vertical velocity together with the close distance to the Galactic plane (Fig. 4) indicate that the cluster will spend its time near the Galactic plane, while the large $U$-velocity component, indicates a radial orbit. We note that NGC 6528's distance has a wide range of values, even from more recent determinations (e.g., Feltzing \& Johnson 2002 determine a distance of $7.2 \mathrm{kpc}$, while HR99 give a distance of 9.3-10.7 kpc from isochrone fitting, and from the horizontal-branch metallicity relation, respectively). Even if, according to distance errors, NGC 6528 is displaced from the far side of the bulge to the near side, the $U$-velocity component will remain practically the same (while the $\Pi$ component changes sign, but $\Theta$ does not, see Fig. 4). This will leave the character of the orbit unchanged.

These kinematical arguments together with the fact that NGC 6528 is, arguably, the 
most metal rich Galactic globular cluster (Feltzing \& Johnson 2002), make a strong case for a globular formed within the bar.

We note that, the two clusters that are located outside the bar limits, NGC 6316 and 6723 (Fig. 4), also have kinematics inconsistent with that of the bar. Among the clusters located within the bar limits, only NGC 6522 - a metal poor cluster — seems to have kinematics inconsistent with that of the bar.

\section{Summary}

We have measured absolute proper motions on the Hipparcos system for four globular clusters located in the bulge region. In addition to these, we include in our discussion three clusters located in the bulge region that had previous proper-motion measurements with respect to bulge stars. We caution that velocities determined from these latter proper motions can be regarded as with respect to an inertial frame, only under strict assumptions for the location of the cluster.

Without a detailed orbital analysis that we defer for a future paper, we discuss the kinematics of these clusters in relation with their metallicities and masses. All of the clusters have velocities smaller than the escape velocity from the bulge, indicating that they have orbits that never leave the bulge region. From the three metal-poor clusters in our sample, two of them, namely NGC 6552, and NGC 6723 have kinematics consistent with halo membership. The third, NGC 6266, seems to belong to a rotationally supported system (reminding one of the thick disk, but not necessarily thick disk at these GC distances) rather than to a pressure supported one. Of the four metal rich clusters, two clusters NGC 6304, and NGC 6553 have kinematics resembling a rotationally supported system, much like NGC 6266. NGC 6528's velocity indicates a radial orbit, confined to the Galactic 
plane. It's rotation velocity is consistent with the velocity predicted from the rotation of a solid body that has the angular pattern speed of the Milky Way bar. As a low-mass, and arguably the most metal-rich Milky Way cluster, NGC 6528 may be a genuine bar cluster. The fourth metal rich cluster, NGC 6316 has kinematics consistent with membership to a hotter system than the bar.

This work is supported in part by NSF grant AST-0098687. 


\section{REFERENCES}

Alvarado, F., Wenderoth, E., Alcaino, G., \& Liller, W. 1994, AJ, 107, 631

Armandroff, T. 1989, AJ97, 375

Athanassoula, E. 2002, ApJ, 569, L83

Barbuy, B., Bica, E., \& Ortolani, S. 1998, A\&A33, 117

Beers, T. C., Drilling, J. S., Rossi, S., Chiba, M., Rhee, J., Fuhrmeister, B., Norris, J. E., \& Hippel, T. 2002, AJ, 124, 931

Bertin, E., \& Arnouts, S. 1996, A\&AS, 117, 393

Bissantz, N., \& Gerhard, O. 2002, MNRAS, 330, 591

Brocato, E, Buonanno, R., Malakhova, Y., \& Piersimoni, A. M. 1996, A\&A, 311, 778

Burkert, A. \& Smith, G. H. 1997, ApJ, 474, L15 (BS97)

Chiba, M. \& Beers, T. 2001, ApJ, 549, 325

Cole, A. A. \& Weinberg, M. D. 2002, ApJ, 574, L43

Côté, P. 1999, AJ, 118, 406

Cudworth, K. M., \& Hanson, R. B. 1993, AJ, 105, 168

Dinescu, D. I., Girard, T. M., van Altena, W. F., Méndez, R., \& López, C. E. 1997, AJ, 114, 1014 (Paper I)

Dinescu, D. I., van Altena, W. F., Girard, T. M., López, C. E. 1999, AJ, 117, 277 (paper II)

Dinescu, D. I., Girard, T. M., \& van Altena, W. F. 1999, AJ, 117, 1792 (Paper III)

Dinescu, D. I., Girard, T. M., \& van Altena, W. F. 2002, in ASP Conf. Ser. ***, New Horizons in Globular Cluster Astronomy, ed. G. Piotto, G. Meylan, G. Djorgovski, \& M. Riello 
ESA 1997, The Hipparcos and Tycho Catalogues (ESA SP-1200) (Noordwijk: ESA)

Feltzing, S., \& Johnson, R. A. 2002, A\&A385, 67

Frenk, C. S. \& White, S. D. M. 1980, MNRAS, 193, 295

Harris, W. E. 1996, AJ, 120, 1892 (H96)

Hartwick, F. D. A. 1987, in The Galaxy; Proceedings of the NATO Advanced Study Institute, Cambridge, England (Dordrecht, D. Reidel Publishing Co., p. 281

Heitsch, F. \& Richtler, T. 1999, A\&A, 1999, 347, 455 (HR99)

Gerhard, O. E. 1996, in Proceedings of the 169th Symposium of the IAU, The Hague, The Netherlands (Dordrecht Kluwer), ed. L. Blitz and P. Teuben, p. 79

Gerhard, O. 2002, in ASP Conf. Ser. ***, The Dynamics, Structure and History of Galaxies, ed. G. S. DaCosta and E. M. Sadler

Ibata, R. A. \& Gilmore, G. F. 1995, MNRAS, 275, 605

Lasker, B. M., Sturch, C. R., McLean, B. J., Russell, J. L., Jenker, H., \& Shara, M. 1990, AJ, 99, 2019

Monet, D., Bird, A., Canzian, B., Dahn, C., Guetter, H., Harris, H., Henden, A., Levine, S., Luginbuhl, C., Monet, A. K. B., Rhodes, A., Riepe, B., Sell, S., Stone, R., Vrba, F \& Walker, R 1998, The USNO-A2.0 Catalogue, (U.S. Naval Observatory, Washington $\mathrm{DC})$

Minniti, D. 1995, AJ, 109, 1663

Minniti, D. 1996, ApJ, 459, 579

Pfenniger, D. 1999, in The Formation of Galactic Bulges, ed. C. M. Carollo, H. C. Ferguson, and R. F. G. Wyse (Cambridge University Press), p. 95

Pfenniger, D. \& Friedli, D. 1991, A\&A, 252, 75 
Ortolani, S, Momany, Y., \& Barbuy, B. 2000, A\&A, 357, 495

Platais, I., Girard, T. M., Kozhurina-Platais, V., van Altena, W. F., López, C. E., Méndez, R., Ma, W-s., Yang, T-g., MacGillivray, H. T., \& Yentis, D. J. 1998, AJ, 116, 2556

Ratnatunga, K. U., Bahcall, J. N., \& Casertano, S. 1989, ApJ, 291, 260

Rosenberg, A., Piotto, G., Saviane, I., \& Aparicio, A. 2000, A\&AS, 144, 5

Siegel, M., Mjewski, S. R., Reid, I. N., \& Thompson, I. B. 2002, ApJ, 578, 151

Stanek, K. Z., Udalski, A., Szymanski, M., Kaluzny, J., Kubiak, M., Mateo, M., \& Krzeminski, W. 1997, ApJ, 477, 163

Terndrup, D., Popowski, P., Gould, A., Rich, R. M., \& Sadler, E. M. 1998, AJ, 115, 1476

Tiede, G. P., \& Terndrup, D. M. 1999, AJ, 118, 895

Weiner, B. J. \& Sellwood, J. A. 1999, ApJ, 524, 112

Zacharias, N., Urban, S. E., Zacharias, M. I., Hall, D. M., Wycoff, G. L., Rafferty, T. J., Germain, M. E., Holdenried, E. R., Pohlman, J. W., Gauss, F. S., Monet, D. G., \& Winter, L. 2000, AJ, 120, 2131 (Z00)

Zinn, R. 1985, ApJ, 293, 424

Zinn, R. 1996, in ASP Conf. Ser. 92, Formation of the Galactic Halo...Inside and Out, ed. H. Morrison, \& A. Sarajedini (San Francisco: ASP), 211

Zoccali, M., Renzini, A., Ortolani, S., Bica, E. \& Barbuy, B. 2001, AJ121, 2638 
Fig. 1.- The correction to absolute proper motion for plate-pair solutions 506Y1Y2 and 506y1-ucac, left and right panel respectively. Labels represent the various image orders as described in the text. The closed symbol is the weighted mean of the measurements.

Fig. 2.- Relative proper motions of stars in the region of NGC 6304 as a function of magnitude, for visual-plate solutions in SPM fields 506 and 507. Horizontal lines represent the (centering-error) weighted means.

Fig. 3.- Absolute proper-motion results for each cluster. Open symbols show the various plate-pair solutions, and the closed symbols represent the final adopted absolute proper motion.

Fig. 4.- Projection on to the Galactic plane (top) and perpendicular to the Galactic plane (bottom) of the positions and velocities of the clusters. Open symbols are the metal poor clusters, while closed symbols are the metal rich clusters. The ellipse represents the Galactic bar with geometric parameters taken from Bissantz \& Gerhard (2002). The GC is represented with a cross, and the dotted circle in the top panel marks a radius of $2.7 \mathrm{kpc}$, as an indication of the bulge region. 
TABLE 1. Plate-pair Solutions

TABLE 2. Absolute Proper Motion Results

TABLE 3. Cluster Data

TABLE 4. Velocities

TABLE 5. Cluster Parameters 
Table 1. Plate-pair Solutions

\begin{tabular}{|c|c|c|}
\hline NGC & Plate-pair solution & Comments \\
\hline 6626 & 506B1B2, 506Y1Y2, 506B1-ucac, 506Y1-ucac & \\
\hline 6304 & 506B1B2, 506Y1Y2, 506B1-ucac, 506Y1-ucac & dompr amulai n 507D1 \\
\hline 6316 & $506 Y 1 Y 2,507 Y 1 Y 2$ & cluster stars are too faint for UCAC1 \\
\hline 6723 & 440B1B2, 440Y1B2, 440B1-ucac, 440Y1-ucac & there is no visual 2nd-epoch SPM plate \\
\hline
\end{tabular}


Table 4. Velocities

\begin{tabular}{rrrrrrrrrr}
\hline \hline NGC & $\mathrm{X}$ & $\begin{array}{c}\mathrm{Y} \\
(\mathrm{kpc})\end{array}$ & $\mathrm{Z}$ & $U$ & $\begin{array}{c}V^{\prime} \\
\left(\mathrm{km} \mathrm{s}^{-1}\right)\end{array}$ & $W$ & $\Pi$ & $\begin{array}{c}\Theta \\
\left(\mathrm{km} \mathrm{s}^{-1}\right)\end{array}$ & $\Omega_{b} * R_{G C}$ \\
\hline 6266 & 1.2 & -0.8 & 0.9 & $78(03)$ & $152(15)$ & $72(14)$ & $-17(09)$ & $170(13)$ & 125 \\
6304 & 1.9 & -0.4 & 0.6 & $105(04)$ & $162(12)$ & $32(09)$ & $66(05)$ & $181(11)$ & 117 \\
6316 & -2.9 & -0.5 & 1.1 & $-70(08)$ & $85(34)$ & $66(32)$ & $53(11)$ & $-96(33)$ & 177 \\
6723 & -0.4 & 0.0 & -2.6 & $87(07)$ & $147(22)$ & $10(18)$ & $-83(06)^{\dagger}$ & $-150(22)^{\dagger}$ & 24 \\
& & & & & & & \\
6522 & 0.2 & 0.1 & -0.5 & $27(04)$ & $57(09)$ & $-227(25)$ & $53(06)^{\dagger}$ & $34(08)^{\dagger}$ & 13 \\
6528 & -1.1 & 0.2 & -0.7 & $-197(04)$ & $-26(11)$ & $4(10)$ & $190(04)$ & $58(11)$ & 67 \\
6553 & 2.4 & 0.5 & -0.3 & $9(03)$ & $225(17)$ & $14(02)$ & $55(05)$ & $219(16)$ & 147 \\
\hline
\end{tabular}
and possibly misleading. 
Table 5. Cluster Parameters

\begin{tabular}{|c|c|c|c|c|c|}
\hline NGC & {$[\mathrm{Fe} / \mathrm{H}]$} & $\mathrm{M}_{V}$ & $\begin{array}{l}\mathrm{V}_{t} \\
(\mathrm{~km}\end{array}$ & $\begin{array}{l}V_{e s c, b} \\
\left.\mathrm{~s}^{-1}\right)\end{array}$ & $\begin{array}{c}\Psi \\
\left(^{\circ}\right)\end{array}$ \\
\hline 6266 & -1.29 & -9.19 & 185 & 349 & 38 \\
\hline 6304 & -0.59 & -7.32 & 195 & 329 & 17 \\
\hline 6316 & -0.55 & -8.35 & 128 & 277 & 31 \\
\hline 6723 & -1.12 & -7.86 & 172 & 298 & 82 \\
\hline 6522 & -1.44 & -7.67 & 236 & 474 & 77 \\
\hline 6528 & -0.17 & -6.93 & 199 & 382 & 66 \\
\hline 6553 & -0.34 & -7.99 & 226 & 302 & 08 \\
\hline
\end{tabular}

